\title{
AN ASYMPTOTIC EXPANSION OF SELBERG'S CENTRAL LIMIT THEOREM NEAR THE CRITICAL LINE
}

\author{
YOONBOK LEE
}

\begin{abstract}
We find an asymptotic expansion of Selberg's central limit theorem for the Riemann zeta function on $\sigma=\frac{1}{2}+(\log T)^{-\theta}$ and $t \in[T, 2 T]$, where $0<\theta<\frac{1}{2}$ is a constant.
\end{abstract}

\section{INTRODUCTION}

Let $\theta>0$ and $\sigma_{T}:=\sigma_{T}(\theta)=\frac{1}{2}+(\log T)^{-\theta}$ throughout the paper. Selberg's central limit theorem (Theorem 2 in [10]) says that for $\frac{1}{2} \leq \sigma \leq \sigma_{T}$, the function

$$
\frac{\log \zeta(\sigma+i t)}{\sqrt{\pi \sum_{p<t} p^{-2 \sigma}}}
$$

has a normal Gaussian distribution in the complex plane. Note that

$$
\sum_{p<t} p^{-2 \sigma}=\log \left(\min \left(\frac{1}{\sigma-1 / 2}, \log t\right)\right)+O(1)
$$

for $\sigma \geq \frac{1}{2}$. Recently, Radziwiłł and Soundararajan in [9] provide a simple proof of Selberg's central limit theorem for $\log \left|\zeta\left(\frac{1}{2}+i t\right)\right|$.

When $\sigma>1 / 2$ is not too close to $1 / 2, \log \zeta(\sigma+i t)$ has a nice approximation by a Dirichlet polynomial so that allows us to improve Selberg's central limit theorem by finding lower order terms. In this direction, Ha and Lee in [3] prove the following theorem.

Theorem 1.1 (Corollary 1.5 of [3]). Let $0<\theta<\frac{1}{2}, a<b$ and $c<d$ be real numbers. There exist polynomials $g_{k}(x, y)$ of degree $\leq k$ such that

$$
\begin{aligned}
\frac{1}{T} \operatorname{meas}\{t & \left.\in[T, 2 T]: \frac{\log \zeta\left(\sigma_{T}+i t\right)}{\sqrt{\pi \psi_{T}}} \in[a, b] \times[c, d]\right\} \\
& =\sum_{0 \leq k \leq 5} \frac{1}{{\sqrt{\psi_{T}}}^{k}} \int_{c}^{d} \int_{a}^{b} g_{k}(x, y) e^{-\pi\left(x^{2}+y^{2}\right)} d x d y+O\left(\frac{1}{(\log \log T)^{3}}\right)
\end{aligned}
$$

as $T \rightarrow \infty$, where $g_{0}(x, y)=1$ and

$$
\psi_{T}:=\sum_{p} \sum_{k \geq 1} \frac{1}{k^{2}} p^{-2 k \sigma_{T}}=\theta \log \log T+O(1) .
$$

Our main theorem improves it by finding lower order terms and expressing the integral of (1.1) in terms of Hermite polynomials.

Date: June 4, 2021.

2010 Mathematics Subject Classification. 11M06.

Key words and phrases. Selberg's central limit theorem, Riemann zeta function. 
Theorem 1.2. Let $0<\theta<\frac{1}{2}, a<b$ and $c<d$ be real numbers. There exist constants $\epsilon, \eta>0$ and a sequence $\left\{d_{k, \ell}\right\}_{k, \ell \geq 0}$ of real numbers such that

$$
\begin{aligned}
\frac{1}{T} \operatorname{meas}\{t & \left.\in[T, 2 T]: \frac{\log \zeta\left(\sigma_{T}+i t\right)}{\sqrt{\pi \psi_{T}}} \in[a, b] \times[c, d]\right\} \\
= & \sum_{k+\ell \leq \epsilon \psi_{T}} \frac{d_{k, \ell}}{{\sqrt{\psi_{T}}}^{k+\ell}} \int_{c}^{d} \int_{a}^{b} e^{-\pi\left(x^{2}+y^{2}\right)} H_{k}(\sqrt{\pi} x) H_{\ell}(\sqrt{\pi} y) d x d y+O\left(\frac{1}{(\log T)^{\eta}}\right)
\end{aligned}
$$

as $T \rightarrow \infty$, where $H_{n}(x)$ is the $n$-th Hermite polynomial defined by

$$
H_{n}(x):=(-1)^{n} e^{x^{2}} \frac{d^{n}}{d x^{n}}\left(e^{-x^{2}}\right) .
$$

Moreover, $d_{0,0}=1$ and $d_{k, \ell}=0$ for $k+\ell=1,2$.

The sequence $\left\{d_{k, \ell}\right\}$ is defined by its generating function in (2.19). Since $d_{k, \ell}=0$ for $k+\ell=1,2$ in Theorem 1.2, we have the following corollary.

Corollary 1.3. Let $0<\theta<\frac{1}{2}, a<b$ and $c<d$ be real numbers, then we have

$\frac{1}{T} \operatorname{meas}\left\{t \in[T, 2 T]: \frac{\log \zeta\left(\sigma_{T}+i t\right)}{\sqrt{\pi \psi_{T}}} \in[a, b] \times[c, d]\right\}=\int_{c}^{d} \int_{a}^{b} e^{-\pi\left(x^{2}+y^{2}\right)} d x d y+O\left(\frac{1}{(\log \log T)^{3 / 2}}\right)$.

Remark that Hejhal in [5, Theorem 2.1] extends Theorem 1.2 to a multi-dimensional setting. For a verification, we provide a useful identity

$$
\begin{aligned}
\sqrt{\pi} \int_{x_{1}}^{x_{2}} e^{-\pi x^{2}} H_{n+1}(\sqrt{\pi} x) d x & =e^{-\pi x_{1}^{2}} H_{n}\left(\sqrt{\pi} x_{1}\right)-e^{-\pi x_{2}^{2}} H_{n}\left(\sqrt{\pi} x_{2}\right) \\
& =(-\sqrt{\pi})^{-n}\left(\Phi^{(n+1)}\left(x_{1}\right)-\Phi^{(n+1)}\left(x_{2}\right)\right)
\end{aligned}
$$

for $n \geq 0$, where $\Phi(x):=\int_{0}^{x} e^{-\pi u^{2}} d u$ is defined in [5]. However, this paper is still meaningful, since there is only a sketched proof in [5] and the asymptotic expansion in our theorem is of length $\sim \epsilon \theta \log \log T$, while the expansion in [5] is of any constant length.

We also remark that the expansion in Theorem 1.2 is similar to an Edgeworth expansion in the probability theory. For further information, see Chapter 7 of [2].

We prove Theorem 1.2 at the end of Section 2 .

\section{Estimates ON THE RANDOM MODEL}

The random Riemann zeta function is defined by the product

$$
\zeta(\sigma, X):=\prod_{p}\left(1-\frac{X(p)}{p^{\sigma}}\right)^{-1},
$$

where $X(p)$ is independent and identically distributed random variables on the unit circle $|z|=1$ assigned for each prime $p$. The product converges almost surely for $\sigma>\frac{1}{2}$. If $\sigma>\frac{1}{2}$ is not too close to $\frac{1}{2}$, then the distribution of the random model $\log \zeta(\sigma, X)$ approximates that of $\log \zeta(\sigma+i t)$. More precisely, the discrepancy defined by

$$
D_{\sigma}(T):=\sup _{\mathcal{R}}\left|\frac{1}{T} \operatorname{meas}\{t \in[T, 2 T]: \log \zeta(\sigma+i t) \in \mathcal{R}\}-\mathbb{P}[\log \zeta(\sigma, X) \in \mathcal{R}]\right|
$$


is small for $\sigma \geq \sigma_{T}$, where the supremum is taken over rectangles $\mathcal{R}$ with sides parallel to the coordinate axes. Lamzouri, Lester and Radziwiłł in [6] show that

$$
D_{\sigma}(T)=O\left(\frac{1}{(\log T)^{\sigma}}\right)
$$

holds for fixed $\sigma>\frac{1}{2}$, which improves earlier results of Matsumoto [7], 8] and Matsumoto and Harman [4]. By the same method, Ha and Lee in [3] show that for each $0<\theta<\frac{1}{2}$, there is a constant $\eta>0$ such that

$$
D_{\sigma_{T}}(T)=O_{\eta}\left(\frac{1}{(\log T)^{\eta}}\right)
$$

Define

$$
\Phi_{\text {rand }}(\mathcal{B}):=\mathbb{P}\left[\log \zeta\left(\sigma_{T}, X\right) \in \mathcal{B}\right]
$$

for a Borel set $\mathcal{B} \subset \mathbb{C}$. It is known that this measure has a density function $F_{\sigma_{T}}$ such that

$$
\mathbb{P}\left[\log \zeta\left(\sigma_{T}, X\right) \in \mathcal{B}\right]=\iint_{\mathcal{B}} F_{\sigma_{T}}(x, y) d x d y
$$

holds for any region $\mathcal{B}$. For a proof, see [1, Theorem 11] or [3, Proposition 3.1]. Since we have

$$
\begin{aligned}
& \frac{1}{T} \operatorname{meas}\{t\left.\in[T, 2 T]: \frac{\log \zeta\left(\sigma_{T}+i t\right)}{\sqrt{\pi \psi_{T}}} \in[a, b] \times[c, d]\right\} \\
&=\mathbb{P}\left[\frac{\log \zeta\left(\sigma_{T}, X\right)}{\sqrt{\pi \psi_{T}}} \in[a, b] \times[c, d]\right]+O\left(\frac{1}{(\log T)^{\eta}}\right) \\
&=\int_{c \sqrt{\pi \psi_{T}}}^{d \sqrt{\pi \psi_{T}}} \int_{a \sqrt{\pi \psi_{T}}}^{b \sqrt{\pi \psi_{T}}} F_{\sigma_{T}}(x, y) d x d y+O\left(\frac{1}{(\log T)^{\eta}}\right)
\end{aligned}
$$

by (2.2) and (2.3), it is enough to find an asymptotic for $F_{\sigma_{T}}(x, y)$ to prove Theorem 1.2 Since we have

$$
F_{\sigma_{T}}(x, y)=\iint_{\mathbb{R}^{2}} \widehat{\Phi}_{\text {rand }}(u, v) e^{-2 \pi i(u x+v y)} d u d v
$$

by the Fourier inversion, we next estimate the Fourier transform

$$
\widehat{\Phi}_{\text {rand }}(u, v)=\mathbb{E}\left[e^{2 \pi i\left(u \operatorname{Re}\left(\log \zeta\left(\sigma_{T}, X\right)\right)+v \operatorname{Im}\left(\log \zeta\left(\sigma_{T}, X\right)\right)\right.}\right] .
$$

By (2.1) we have

$$
\widehat{\Phi}_{\mathrm{rand}}(u, v)=\prod_{p} J\left(\pi u, \pi v, p^{-\sigma_{T}}\right),
$$

where

$$
J(u, v, w):=\mathbb{E}\left[e^{-2 i(u \operatorname{Re} \log (1-w X)+v \operatorname{Im} \log (1-w X))}\right] .
$$

Then we have the following lemma, which is a modification of [3, Lemma 3.3].

Lemma 2.1. Let $0<r<1$ and $C_{r}=-\frac{1}{r} \log (1-r)$. Then we have series expansions

$$
J(u, v, w)=1+\sum_{k, \ell \geq 1} \frac{i^{k+\ell}}{k ! \ell !} a_{k, \ell}(w)(u+i v)^{k}(u-i v)^{\ell}
$$


for any $u, v \in \mathbb{R}$ and $0<w<1$, and

$$
\log J(u, v, w)=\sum_{k, \ell \geq 1} \frac{i^{k+\ell}}{k ! \ell !} b_{k, \ell}(w)(u+i v)^{k}(u-i v)^{\ell}
$$

for $u^{2}+v^{2} \leq\left(2 r C_{r}\right)^{-2}$ and $|w| \leq r$, where the coefficients $a_{k, \ell}(w)$ and $b_{k, \ell}(w)$ are defined by

$$
\begin{aligned}
a_{k, \ell}(w) & =\sum_{n \geq \max (k, \ell)}\left(\sum_{\substack{n_{1}+\cdots+n_{k}=n \\
n_{i} \geq 1}} \frac{1}{n_{1} \cdots n_{k}}\right)\left(\sum_{\substack{m_{1}+\cdots+m_{\ell}=n \\
m_{i} \geq 1}} \frac{1}{m_{1} \cdots m_{\ell}}\right) w^{2 n}, \\
b_{k, \ell}(w) & =\sum_{n \leq \min (k, \ell)} \frac{(-1)^{n-1}}{n} \sum_{\substack{k_{1}+\cdots k_{n}=k \\
\ell_{1}+\cdots+\ell_{n}=\ell \\
k_{i}, \ell_{i} \geq 1}}\left(\begin{array}{c}
k \\
k_{1}, \ldots, k_{n}
\end{array}\right)\left(\begin{array}{c}
\ell \\
\ell_{1}, \ldots, \ell_{n}
\end{array}\right) a_{k_{1}, \ell_{1}}(w) \cdots a_{k_{n}, \ell_{n}}(w) .
\end{aligned}
$$

Moreover, we have

(1) $b_{k, \ell}(w)$ is real and $b_{1,1}(w)=\sum_{m \geq 1} \frac{1}{m^{2}} w^{2 m}$,

(2) $a_{k, \ell}(w), b_{k, \ell}(w) \ll_{k, \ell} w^{2 \max (k, \ell)}$,

(3) $a_{k, \ell}(w)=a_{\ell, k}(w)$ and $b_{k, \ell}(w)=b_{\ell, k}(w)$,

(4) $0<a_{k, \ell}(w) \leq C_{r}^{k+\ell} w^{k+\ell}$ and $\left|b_{k, \ell}(w)\right| \leq C_{r}^{k+\ell} \min (k, \ell)^{k+\ell} w^{k+\ell}$ for $0<w \leq r$.

Proof. The lemma is basically Lemma 3.3 of [3]. (See [3, page 852, lines 10 and 20] for (2.8) and (2.9).) The condition $b_{k, \ell}(w)=b_{\ell, k}(w)$ is the only statement, which is not proved therein. However, this easily follows from (2.9) and the fact that $a_{k, \ell}(w)=a_{\ell, k}(w)$.

Lemma 2.2. Let $\psi_{T}$ be as in (1.2). Define

$$
\tilde{b}_{k, \ell}:=\frac{(\pi i)^{k+\ell}}{k ! \ell !} \sum_{p} b_{k, \ell}\left(p^{-1 / 2}\right)
$$

where $b_{k, \ell}(w)$ is defined in (2.9). Then there is a constant $\delta_{1}>0$

$$
\sum_{p} \log J\left(\pi u, \pi v, p^{-\sigma_{T}}\right)=-\pi^{2}\left(u^{2}+v^{2}\right) \psi_{T}+\sum_{\substack{k, \ell \geq 1 \\ k+\ell \geq 3}} \tilde{b}_{k, \ell}(u+i v)^{k}(u-i v)^{\ell}+O\left(\frac{1}{(\log T)^{\theta}}\right)
$$

for $u^{2}+v^{2} \leq \delta_{1}$.

Proof. Since the inequality $1-e^{-x} \leq x$ holds for any $x \geq 0$, we have

$$
0<1-p^{-\frac{2 n}{(\log T)^{\theta}}} \leq \frac{2 n \log p}{(\log T)^{\theta}}
$$

for any prime $p$ and integer $n>0$. For any $\epsilon>0$, there is a constant $C(\epsilon)>0$ such that $\log x \leq C(\epsilon) x^{\epsilon}$ for all $x \geq 1$. Thus, we have

$$
\log p^{n} \leq C(2 \epsilon) p^{2 n \epsilon}
$$


By (2.8), (2.14), (2.15), and (4) of Lemma 2.1, we have

$$
\begin{aligned}
0<a_{k, \ell} & \left(p^{-1 / 2}\right)-a_{k, \ell}\left(p^{-\sigma_{T}}\right) \\
& \leq \frac{2 C(2 \epsilon)}{(\log T)^{\theta}} \sum_{n \geq \max (k, \ell)}\left(\sum_{\substack{n_{1}+\cdots+n_{k}=n \\
n_{i} \geq 1}} \frac{1}{n_{1} \cdots n_{k}}\right)\left(\sum_{\substack{m_{1}+\cdots+m_{\ell}=n \\
m_{i} \geq 1}} \frac{1}{m_{1} \cdots m_{\ell}}\right) \frac{1}{p^{(1-2 \epsilon) n}} \\
& =\frac{2 C(2 \epsilon)}{(\log T)^{\theta}} a_{k, \ell}\left(p^{-1 / 2+\epsilon}\right) \leq \frac{2 C(2 \epsilon)}{(\log T)^{\theta}} C_{r}^{k+\ell} p^{-(1 / 2-\epsilon)(k+\ell)}
\end{aligned}
$$

for $k, \ell \geq 1$, any prime $p$ and any $\epsilon>0$ with a choice $r=2^{-1 / 2+\epsilon}$. Since

$$
\prod_{j=1}^{n} x_{j}-\prod_{j=1}^{n} y_{j}=\sum_{j=1}^{n}\left(\prod_{i \geq j} x_{i} \prod_{i<j} y_{i}-\prod_{i>j} x_{i} \prod_{i \leq j} y_{i}\right)
$$

we have

$$
\begin{aligned}
& 0<\prod_{j=1}^{n} a_{k_{j}, \ell_{j}}\left(p^{-1 / 2}\right)-\prod_{j=1}^{n} a_{k_{j}, \ell_{j}}\left(p^{-\sigma_{T}}\right) \leq \sum_{j=1}^{n}\left(a_{k_{j}, \ell_{j}}\left(p^{-1 / 2}\right)-a_{k_{j}, \ell_{j}}\left(p^{-\sigma_{T}}\right)\right) \prod_{i \neq j} a_{k_{i}, \ell_{i}}\left(p^{-1 / 2}\right) \\
& \quad \leq \sum_{j=1}^{n} \frac{2 C(2 \epsilon)}{(\log T)^{\theta}} C_{r}^{k_{j}+\ell_{j}} p^{-(1 / 2-\epsilon)\left(k_{j}+\ell_{j}\right)} \prod_{i \neq j} C_{r}^{k_{i}+\ell_{i}} p^{-1 / 2\left(k_{i}+\ell_{i}\right)} \\
& \quad \leq n \frac{2 C(2 \epsilon)}{(\log T)^{\theta}} C_{r}^{\sum_{j}\left(k_{j}+\ell_{j}\right)} p^{-(1 / 2-\epsilon) \sum_{j}\left(k_{j}+\ell_{j}\right)}
\end{aligned}
$$

by (2.13) and (4) of Lemma 2.1. The equation (2.9) and the above inequality imply that

$$
\begin{aligned}
& \left|b_{k, \ell}\left(p^{-1 / 2}\right)-b_{k, \ell}\left(p^{-\sigma_{T}}\right)\right| \\
& \quad \leq \sum_{n \leq \min (k, \ell)} \frac{1}{n} \sum_{\substack{k_{1}+\cdots k_{n}=k \\
\ell_{1}+\cdots+\ell_{n}=\ell \\
k_{i}, \ell_{i} \geq 1}}\left(\begin{array}{c}
k \\
k_{1}, \ldots, k_{n}
\end{array}\right)\left(\begin{array}{c}
\ell \\
\ell_{1}, \ldots, \ell_{n}
\end{array}\right)\left|\prod_{j=1}^{n} a_{k_{j}, \ell_{j}}\left(p^{-1 / 2}\right)-\prod_{j=1}^{n} a_{k_{j}, \ell_{j}}\left(p^{-\sigma_{T}}\right)\right| \\
& \quad \leq \sum_{n \leq \min (k, \ell)} n^{k+\ell} \frac{2 C(2 \epsilon)}{(\log T)^{\theta}} C_{r}^{k+\ell} p^{-(1 / 2-\epsilon)(k+\ell)} \\
& \leq \frac{3 C(2 \epsilon)}{(\log T)^{\theta}}(\min (k, \ell))^{k+\ell} C_{r}^{k+\ell} p^{-(1 / 2-\epsilon)(k+\ell)}
\end{aligned}
$$

By Stirling's formula and the above inequality with $0<\epsilon<\frac{1}{6}$, we have

$$
\begin{aligned}
& \sum_{p} \sum_{k+\ell \geq 3} \frac{\pi^{k+\ell}\left(u^{2}+v^{2}\right)^{(k+\ell) / 2}}{k ! \ell !}\left|b_{k, \ell}\left(p^{-1 / 2}\right)-b_{k, \ell}\left(p^{-\sigma_{T}}\right)\right| \\
& \ll \frac{1}{(\log T)^{\theta}} \sum_{p} \sum_{k+\ell \geq 3}\left(\frac{\pi \sqrt{\delta_{1}} e C_{r}}{p^{1 / 2-\epsilon}}\right)^{k+\ell} \ll \frac{1}{(\log T)^{\theta}}
\end{aligned}
$$


for $u^{2}+v^{2} \leq \delta_{1}$, where $\delta_{1}$ is a constant satisfying $\frac{\pi \sqrt{\delta_{1}} e C_{r}}{2^{1 / 2}-\epsilon}<1$. By (2.7), we have

$$
\sum_{p} \log J\left(\pi u, \pi v, p^{-\sigma_{T}}\right)=\sum_{k, \ell \geq 1} \frac{(\pi i)^{k+\ell}}{k ! \ell !}(u+i v)^{k}(u-i v)^{\ell} \sum_{p} b_{k, \ell}\left(p^{-\sigma_{T}}\right)
$$

for $u^{2}+v^{2} \leq \delta_{1}$ if $\delta_{1} \leq\left(\pi \sqrt{2} C_{1 / \sqrt{2}}\right)^{-2}$. By (2.14), (2.15) and the identity $\psi_{T}=\sum_{p} b_{1,1}\left(p^{-\sigma_{T}}\right)$, the lemma follows.

Lemma 2.3. There are constants $\delta_{2}, \delta_{3}>0$ and a sequence $\left\{d_{k, \ell}\right\}_{k, \ell \geq 0}$ of real numbers such that

$$
\widehat{\Phi}_{\text {rand }}(u, v)=e^{-\pi^{2}\left(u^{2}+v^{2}\right) \psi_{T}}\left(\sum_{k, \ell \geq 0}(2 \pi i)^{k+\ell} d_{k, \ell} u^{k} v^{\ell}+O\left(\frac{1}{(\log T)^{\theta}}\right)\right)
$$

for $u^{2}+v^{2} \leq \delta_{2}$, where $d_{0,0}=1, d_{k, \ell}=0$ for $k+\ell=1,2$ and $d_{k, \ell}=O\left(\delta_{3}^{-(k+\ell)}\right)$ for $k+\ell \geq 3$.

Proof. By (2.6) and Lemma 2.2, we have

$$
\widehat{\Phi}_{\text {rand }}(u, v)=e^{-\pi^{2}\left(u^{2}+v^{2}\right) \psi_{T}} g(u, v)\left(1+O\left(\frac{1}{(\log T)^{\theta}}\right)\right)
$$

for $u^{2}+v^{2} \leq \delta_{1}$, where

$$
g(u, v):=\exp \left(\sum_{\substack{k, \ell \geq 1 \\ k+\ell \geq 3}} \tilde{b}_{k, \ell}(u+i v)^{k}(u-i v)^{\ell}\right) .
$$

By (2.10), Lemma 2.1 and Stirling's formula, the sum

$$
\begin{aligned}
\sum_{\substack{k, \ell \geq 1 \\
k+\ell \geq 3}}\left|\tilde{b}_{k, \ell}\right|\left|(u+i v)^{k}(u-i v)^{\ell}\right| & \leq \sum_{\substack{k, \ell \geq 1 \\
k+\ell \geq 3}} \frac{\left(\pi \sqrt{u^{2}+v^{2}}\right)^{k+\ell}}{k ! \ell !} \sum_{p} C_{1 / \sqrt{2}}^{k+\ell} \min (k, \ell)^{k+\ell} p^{-(k+\ell) / 2} \\
& \ll \sum_{p} \sum_{\substack{k, \ell \geq 1 \\
k+\ell \geq 3}}\left(\frac{C_{1 / \sqrt{2}} \pi e \sqrt{u^{2}+v^{2}}}{\sqrt{p}}\right)^{k+\ell}
\end{aligned}
$$

is convergent and bounded for $u^{2}+v^{2} \leq \delta_{2}$ provided that $C_{1 / \sqrt{2}} \pi e \sqrt{\delta_{2}}<\sqrt{2}$. Thus, we can find a power series expansion of $g(u, v)$ for $u^{2}+v^{2} \leq \delta_{2}$.

Let $b_{k, \ell}^{\prime}=\tilde{b}_{k, \ell}(2 \pi i)^{-k-\ell}$, then we see that

$$
g\left(\frac{x}{2 \pi i}, \frac{y}{2 \pi i}\right)=\exp \left(\sum_{\substack{k, \ell \geq 1 \\ k+\ell \geq 3}} b_{k, \ell}^{\prime}(x+i y)^{k}(x-i y)^{\ell}\right) .
$$

Since

$$
b_{k, \ell}^{\prime}=\frac{1}{2^{k+\ell} k ! \ell !} \sum_{p} b_{k, \ell}\left(p^{-1 / 2}\right)
$$

by (2.10), we have that $b_{k, \ell}^{\prime}=b_{\ell, k}^{\prime}$ and $b_{k, \ell}^{\prime}$ is real for every $k, \ell$ by Lemma 2.1. Since

$$
\sum_{\substack{k, \ell \geq 1 \\ k+\ell \geq 3}} b_{k, \ell}^{\prime}(x+i y)^{k}(x-i y)^{\ell}-\sum_{\substack{k, \ell \geq 1 \\ k+\ell \geq 3}} \overline{b_{k, \ell}^{\prime}(x+i y)^{k}(x-i y)^{\ell}}
$$




$$
\begin{aligned}
& =\sum_{\substack{k, \ell \geq 1 \\
k+\ell \geq 3}} b_{k, \ell}^{\prime}(x+i y)^{k}(x-i y)^{\ell}-\sum_{\substack{k, \ell \geq 1 \\
k+\ell \geq 3}} b_{k, \ell}^{\prime}(x-i y)^{k}(x+i y)^{\ell} \\
& =\sum_{\substack{k, \ell \geq 1 \\
k+\ell \geq 3}} b_{k, \ell}^{\prime}(x+i y)^{k}(x-i y)^{\ell}-\sum_{\substack{k, \ell \geq 1 \\
k+\ell \geq 3}} b_{\ell, k}^{\prime}(x-i y)^{k}(x+i y)^{\ell}=0,
\end{aligned}
$$

the sum $\sum_{\substack{k, \ell \geq 1 \\ k+\ell \geq 3}} b_{k, \ell}^{\prime}(x+i y)^{k}(x-i y)^{\ell}$ is a power series in $x$ and $y$ with real coefficients. Therefore, there is a sequence $\left\{d_{k, \ell}\right\}_{k, \ell \geq 0}$ of real numbers such that

$$
\sum_{k, \ell \geq 0} d_{k, \ell} x^{k} y^{\ell}:=\exp \left(\sum_{\substack{k, \ell \geq 1 \\ k+\ell \geq 3}} b_{k, \ell}^{\prime}(x+i y)^{k}(x-i y)^{\ell}\right) \text {. }
$$

By (2.18) and (2.19), we have

$$
g(u, v)=\sum_{k, \ell \geq 0}(2 \pi i)^{k+\ell} d_{k, \ell} u^{k} v^{\ell} .
$$

This proves (2.16).

By expanding the right hand side of (2.19), it is easy to see that $d_{0,0}=1$ and $d_{k, \ell}=0$ for $k+\ell=1,2$. Let $\delta_{3}$ be a constant such that $0<\delta_{3}<\frac{\sqrt{2}}{e C_{1 / \sqrt{2}}}$. Since $g(u, v)$ is bounded for $|u|,|v| \leq \frac{\delta_{3}}{2 \pi}$ similarly to (2.17), we have

$$
d_{k, \ell}=\frac{1}{(2 \pi i)^{k+\ell+2}} \oint_{|u|=\frac{\delta_{3}}{2 \pi}} \oint_{|v|=\frac{\delta_{3}}{2 \pi}} \frac{g(u, v)}{u^{k+1} v^{\ell+1}} d v d u=O\left(\delta_{3}^{-(k+\ell)}\right) .
$$

Lemma 2.4. Let $\left\{d_{k, \ell}\right\}_{k, \ell \geq 0}$ be the sequence of real numbers in Lemma 2.3. There exist constants $\epsilon, \eta>0$ such that

$$
F_{\sigma_{T}}(x, y)=e^{-\left(x^{2}+y^{2}\right) / \psi_{T}} \sum_{k+\ell \leq \epsilon \psi_{T}} \frac{d_{k, \ell}}{\pi{\sqrt{\psi_{T}}}^{k+\ell+2}} H_{k}\left(\frac{x}{\sqrt{\psi_{T}}}\right) H_{\ell}\left(\frac{y}{\sqrt{\psi_{T}}}\right)+O\left(\frac{1}{(\log T)^{\eta}}\right)
$$

for all $x, y \in \mathbb{R}$, where $H_{n}(x)$ is the $n$-th Hermite polynomial defined in (1.3).

Proof. Let $\delta_{4}$ be a constant satisfying $0<\delta_{4}<\min \left(\delta_{2}, \delta_{3}^{2}(2 \pi)^{-2}\right)$. By applying Lemma 3.5 of [3] to (2.5), there is a constant $\eta_{1}>0$ such that

$$
F_{\sigma_{T}}(x, y)=\iint_{u^{2}+v^{2} \leq \delta_{4}} \widehat{\Phi}_{\text {rand }}(u, v) e^{-2 \pi i(u x+v y)} d u d v+O\left(\frac{1}{(\log T)^{\eta_{1}}}\right) .
$$

Let $\epsilon$ be a constant satisfying $0<\epsilon<\frac{e}{4} \delta_{2}^{2}$. By Lemma 2.3, we have

$$
\begin{aligned}
F_{\sigma_{T}}(x, y)= & \sum_{k, \ell \geq 0}(2 \pi i)^{k+\ell} d_{k, \ell} \iint_{u^{2}+v^{2} \leq \delta_{4}} e^{-\pi^{2}\left(u^{2}+v^{2}\right) \psi_{T}} u^{k} v^{\ell} e^{-2 \pi i(u x+v y)} d u d v+O\left(\frac{1}{(\log T)^{\eta_{2}}}\right) \\
= & \sum_{k+\ell \leq \epsilon \psi_{T}}(2 \pi i)^{k+\ell} d_{k, \ell} \iint_{u^{2}+v^{2} \leq \delta_{4}} e^{-\pi^{2}\left(u^{2}+v^{2}\right) \psi_{T}} u^{k} v^{\ell} e^{-2 \pi i(u x+v y)} d u d v \\
& +O\left(\sum_{k+\ell>\epsilon \psi_{T}} \frac{(2 \pi)^{k+\ell} \delta_{4}^{(k+\ell) / 2}}{\delta_{3}^{k+\ell} \psi_{T}}+\frac{1}{(\log T)^{\eta_{2}}}\right)
\end{aligned}
$$


where $\eta_{2}=\min \left(\eta_{1}, \theta\right)$. Since $\delta_{4}<\delta_{3}^{2}(2 \pi)^{-2}$, the $O$-term is $O\left((\log T)^{-\eta_{3}}\right)$ for some $\eta_{3}>0$.

To complete the proof, it requires to estimate the last integral, which equals to

$$
\iint_{\mathbb{R}^{2}} e^{-\pi^{2}\left(u^{2}+v^{2}\right) \psi_{T}} u^{k} v^{\ell} e^{-2 \pi i(u x+v y)} d u d v-\iint_{u^{2}+v^{2}>\delta_{4}} e^{-\pi^{2}\left(u^{2}+v^{2}\right) \psi_{T}} u^{k} v^{\ell} e^{-2 \pi i(u x+v y)} d u d v .
$$

The second integral in (2.20) is

$$
\begin{aligned}
\mid \iint_{u^{2}+v^{2}>\delta_{4}} & e^{-\pi^{2}\left(u^{2}+v^{2}\right) \psi_{T}} u^{k} v^{\ell} e^{-2 \pi i(u x+v y)} d u d v \mid \\
& \leq \iint_{u^{2}+v^{2}>\delta_{4}} e^{-\pi^{2}\left(u^{2}+v^{2}\right) \psi_{T}}|u|^{k}|v|^{\ell} d u d v \\
& \leq\left(\iint_{u^{2}+v^{2}>\delta_{4}} e^{-\pi^{2}\left(u^{2}+v^{2}\right) \psi_{T}} d u d v\right)^{1 / 2}\left(\iint_{\mathbb{R}^{2}} e^{-\pi^{2}\left(u^{2}+v^{2}\right) \psi_{T}} u^{2 k} v^{2 \ell} d u d v\right)^{1 / 2} \\
& =e^{-\frac{\pi^{2} \delta_{4}}{2} \psi_{T}} \frac{\sqrt{\pi}}{\left(\pi \sqrt{\psi_{T}}\right)^{k+\ell+2}} \sqrt{\Gamma\left(k+\frac{1}{2}\right) \Gamma\left(\ell+\frac{1}{2}\right)}
\end{aligned}
$$

by the Cauchy-Schwartz inequality. By Stirling's formula, the above is

$$
\ll e^{-\frac{\pi^{2} \delta_{4}}{2} \psi_{T}} \frac{1}{\left(\pi \sqrt{\psi_{T}}\right)^{k+\ell+2}} \frac{(k+1 / 2)^{k / 2}(\ell+1 / 2)^{\ell / 2}}{e^{(k+\ell) / 2}} \leq e^{-\frac{\pi^{2} \delta_{4}}{2} \psi_{T}} \frac{1}{\pi^{2} \psi_{T}}\left(\frac{\sqrt{\epsilon}}{\pi \sqrt{e}}\right)^{k+\ell}
$$

for $k+\ell \leq \epsilon \psi_{T}$. Since $d_{k, \ell}=O\left(\delta_{3}^{-(k+\ell)}\right)$ by Lemma 2.3, the contribution of the second integral in (2.20) to $F_{\sigma_{T}}(x, y)$ is

$$
O\left(\sum_{k+\ell \leq \epsilon \psi_{T}} e^{-\frac{\pi^{2} \delta_{4}}{2} \psi_{T}} \frac{1}{\psi_{T}}\left(\frac{2 \sqrt{\epsilon}}{\delta_{3} \sqrt{e}}\right)^{k+\ell}\right)=O\left(\frac{1}{(\log T)^{\eta_{4}}}\right)
$$

for some $\eta_{4}>0$ since $\epsilon<\frac{e}{4} \delta_{3}^{2}$. Therefore, we have

$$
F_{\sigma_{T}}(x, y)=\sum_{k+\ell \leq \epsilon \psi_{T}}(2 \pi i)^{k+\ell} d_{k, \ell} \iint_{\mathbb{R}^{2}} e^{-\pi^{2}\left(u^{2}+v^{2}\right) \psi_{T}} u^{k} v^{\ell} e^{-2 \pi i(u x+v y)} d u d v+O\left(\frac{1}{(\log T)^{\eta}}\right)
$$

with $\eta=\min \left(\eta_{3}, \eta_{4}\right)$. Since the last integral equals to

$$
\begin{aligned}
& \frac{1}{(-2 \pi i)^{k+\ell}} \frac{\partial^{k+\ell}}{\partial x^{k} \partial y^{\ell}} \iint_{\mathbb{R}^{2}} e^{-\pi^{2}\left(u^{2}+v^{2}\right) \psi_{T}} e^{-2 \pi i(u x+v y)} d u d v \\
= & \frac{1}{(-2 \pi i)^{k+\ell}} \frac{\partial^{k+\ell}}{\partial x^{k} \partial y^{\ell}}\left(\frac{1}{\pi \psi_{T}} e^{-\left(x^{2}+y^{2}\right) / \psi_{T}}\right) \\
= & \frac{1}{\pi \psi_{T}} \frac{1}{\left(2 \pi i \sqrt{\psi_{T}}\right)^{k+\ell}} e^{-\left(x^{2}+y^{2}\right) / \psi_{T}} H_{k}\left(\frac{x}{\sqrt{\psi_{T}}}\right) H_{\ell}\left(\frac{y}{\sqrt{\psi_{T}}}\right),
\end{aligned}
$$

the lemma holds.

Proof of Theorem 1.2. The theorem holds by (2.4) and Lemma 2.4. 


\section{ACKNOWLEDGEMET}

This work was supported by Incheon National University RIBS Grant in 2020. We thank an anonymous referee for informing us of Hejhal's paper [5].

\section{REFERENCES}

[1] V. Borchsenius and B. Jessen, Mean motions and values of the Riemann zeta function, Acta Math. 80 (1948), 97-166.

[2] H. Cramér, Random variables and probability distributions, 3rd edition, Cambridge University Press, 1970.

[3] J. Ha and Y. Lee, The a-values of the Riemann zeta function near the critical line, J. Math. Anal. Appl. 464, (2018), 838-863.

[4] G. Harman and K. Matsumoto, Discrepancy estimates for the value-distribution of the Riemann zetafunction IV, J. London Math. Soc. (2), 50 (1994), 17-24.

[5] D. Hejhal, On Euler products and multi-variate Gaussians, C. R. Acad. Sci. Paris, Ser. I, 337, (2003), $223-226$.

[6] Y. Lamzouri, S. Lester and M. Radziwiłł, Discrepancy bounds for the distribution of the Riemann zetafunction and applications, J. Anal. Math. 139 (2019), no. 2, 453-494.

[7] K. Matsumoto, Discrepancy estimates for the value-distribution of the Riemann zeta-function. I, Acta Arith. 48 (1987), no. 2, 167-190.

[8] K. Matsumoto, Discrepancy estimates for the value-distribution of the Riemann zeta-function. III, Acta Arith. 50 (1988), no. 4, 315-337.

[9] M. Radziwiłł and K. Soundararajan, Selberg's central limit theorem for $\log \left|\zeta\left(\frac{1}{2}+i t\right)\right|$, Enseign. Math. 63 (2017), 1-19.

[10] A. Selberg, Old and new conjectures and results about a class of Dirichlet series, Collected papers vol. $2,47-63$.

Department of Mathematics, Research Institute of Basic Science, Incheon National UniVERSity, 119 ACADEMY-Ro, YeOnsu-Gu, InCHEOn, 22012, Korea

Email address: leeyb@inu.ac.kr, leeyb131@gmail.com 